\title{
Role of Flexible Video Bronchoscopy in Diagnosis of Pulmonary Lesions
}

\author{
Authors \\ Dr Vishal R. More ${ }^{1}$, Dr B.O. Tayade ${ }^{2}$, Dr Sonal S. Arsude ${ }^{3}$ \\ Department of Respiratory Medicine ,NKP Salve Institute of Medical Sciences \& Research Centre, \\ Lata Mangeshkar Hospital, Nagpur, Maharashtra, India \\ Corresponding Author \\ Dr. More Vishal \\ Lata Mangeshkar Hospital, Nagpur PIN 440019 \\ Email: dr.morevishal@gmail.com, Mobile number-7507606889
}

\begin{abstract}
Flexible video bronchoscopy is very useful \& safe procedure for diagnosis of respiratory diseases. It can be performed under local anesthesia in various clinics/hospitals settings providing maximal visualization of tracheobronchial tree. The purpose of this article is to study the utility of Flexible video bronchoscopy in diagnosis of pulmonary lesions and various diagnostic techniques of bronchoscopy. we included cases where radiological \& clinical examination or routine laboratory investigations could not clinch diagnosis \& patients having persistant pulmonary lesions on chest xray in form of collapse, consolidation, nodule or mass. Case record form was maintained. All patients were subjected to sputum \& hematological examination, chest xray, ECG, coagulation profile \& CT scan thorax in some cases. Then patients were subjected to flexible video bronchoscopy. Appropriate samples such as bronchial wash, brushing, biopsy/trans-bronchial needle aspiration (if indicated) were obtained depending on the lesion after thorough evaluation of endobronchial tree. Mean age was $47.3 \pm 18.6$ years. Nearly 2/3rd were males and rest were females. Over half of participants (55.4\%) belonged to rural region. In bronchoscopy, Normal vocal cords were seen in(92.1\%), left fixed cord (6.9\%) \& nodule on left vocal cord (2.0\%). On evaluation after bronchoscopy Pulmonary TB was most common (24.8\%) followed by bacterial pneumonia (24.8\%) and malignancy (23.8\%). Other diagnoses included fungal pneumonia and ILD. Bronchoscopy was nondiagnostic in $22.8 \%$ cases. Patients of respiratory complaints with lesion visible on chest xray who are undiagnosed can derive benefit by flexible bronchoscopy. Use of supportive techniques of bronchial wash, brushing, biopsy aid in diagnosis.
\end{abstract}

\section{INTRODUCTION}

Bronchoscopy, the process of visualization of respiratory tree was first performed by Gustav Killian from Germany in 1887. In early era, the utility of bronchoscopy was limited to therapeutic uses like foreign body removal and strictures dilatation due to tuberculosis, diphtheria infections. Introduction of modern rigid bronchoscopy in early part of 20th century was also restricted to therapeutic uses. Ikeda introduced fiberoptic bronchoscopy (FOB) in late 1960s for primary purpose of evaluating suspected lung malignancy. FOB helps not only in diagnosis but also in disease staging, lesion biopsy and is comfortable to perform, safe and well tolerated ${ }^{(1,2)}$. 
Advancement of FOB to video bronchoscopy provides the added advantage of facilitating teaching and rendering the procedure more interesting for other observers in the bronchoscopy suite ${ }^{(2)}$. Diagnostic yield of FOB is majorly determined by the location of the lesion. In lung malignancy; FOB helps in early detection of lung cancer by providing an avenue for inspection of peripheral airways including those of the upper lobes. FOB provides histological diagnosis in between $60 \%$ to $80 \%$ of cases without serious complications ${ }^{(3,4)}$. FOB is performed for various indications. A large series looking at trends for indication of FOB over 34 years from India reported malignancy $(32.2 \%)$ as most common indication followed by haemoptysis $(11.0 \%)$, tuberculosis (9.5\%) among various other indications ${ }^{(5)}$ In a series of 250 cases from India, bronchoscopy was able to differentiate various lesions from which $26 \%$ were neoplastic, 59\% were non-neoplastic lesions, and no specific pathology in $13 \%$ with $2 \%$ cases having inadequate tissue ${ }^{(6)}$ Besides diagnostic, FOB can be helpful in treatment and monitoring of disease progression. With its expanding use in diagnosis of rare disorders like pulmonary alveolar proteinosis and spindle cell tumour of trachea, it is essential to study indications for bronchoscopy with its yield by different associated methods. In our study we planned to assess the utility of flexible video bronchoscopy in diagnosis of pulmonary lesions at a tertiary care hospital.

\section{AIMS AND OBJECTIVES}

1. To study the utility of Flexible video bronchoscopy in diagnosis of pulmonary lesions.

2. To study utility of various diagnostic techniques of bronchoscopy .

\section{MATERIALS AND METHODS}

We performed a cross sectional study in patients undergoing bronchoscopy at a tertiary care hospital and teaching institute. The methodology adopted in this study is outlined below. Prior to initiation of this study; Institutional ethics committee (IEC) approval to the protocol of the study was obtained.

We conducted this study in the Department of Respiratory Medicine at the tertiary care hospital. It was cross-sectional, observational study. Study duration was from Jan 2015 to August 2016. Sample size was 100.

\section{Inclusion Criteria}

1. Age 18 years and above

2. Clinical, radiological and routine laboratory examination inconclusive

3. Chest X-ray findings of persistent pulmonary lesions in form of collapse, consolidation, nodule, mass or pulmonary infiltrate

4. Patients parents/guardian willing to give informed written consent

\section{Exclusion Criteria}

1. Patients with respiratory failure on ventilators

2. Patients already diagnosed with following diseases

3. Lung cancer.

4. Smear positive pulmonary tuberculosis

5. Recent myocardial infarction.

6. Blood dyscrasias.

\section{Study Intervention}

1.Detailed recording of history. 2. Complete physical and systemic examination. 3. Investigations: $\square$ Complete haemogram. $\square$ Random blood sugar. $\square$ CXR- PA view; Lateral view (if needed) $\square$ Sputum examination for AFB - 2 samples (1 - spot sample \& 1 - early morning sample) carried out by $\mathrm{ZN}$ staining technique. $\square$ Induced sputum for AFB. $\square$ Electrocardiogram (ECG). $\square$ Tests for HIV. $\square$ HBsAg. $\square$ Coagulation profile. $\square$ Ancillary specific investigations (where relevant) o Computed Tomography - thorax. o Mantoux test.

Preparation of the patient: Bronchoscopy was performed in all cases who were recruited into the study. After obtaining well informed written consent, all the bronchoscopies were performed as an elective procedure. Patients were taken up for 
procedures after fasting for 6 to 8 hours. Flexible Video Bronchoscopy (FVB) was done by Bronchoscope of company OLYMPUS BF TYPE $1 T 240$ (Figure 1)

Findings were noted at FVB.

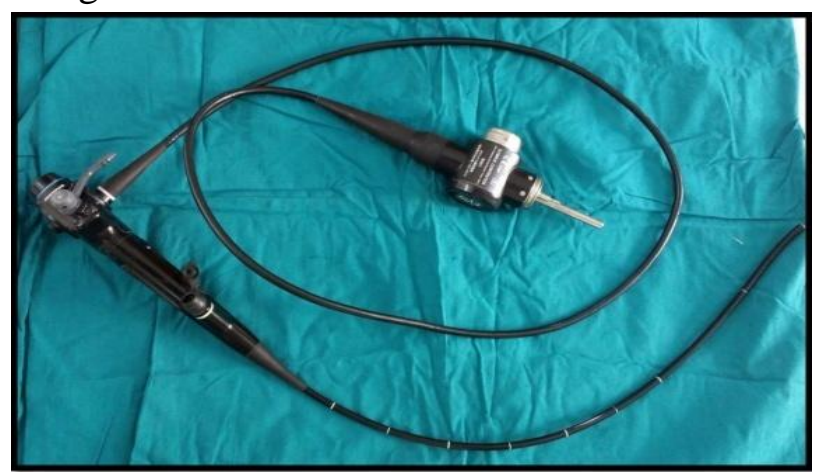

Figure 1 : Showing the Bronchoscope Olympus BF Type 1T240( we used in our study)

Collection and handling of tissue sampling material:

In most patients, various combinations of Bronchial washing, endobronchial biopsy, Bronchial brushing were carried out according to the Bronchoscopic findings. $\square$ When no lesion was seen, Bronchial washing was done in all patients and Bronchial brushing in some patients from the appropriate segments as determined by chest x-ray and/or CT Thorax images

Pathological Examination $\square \quad$ Cytology Histopathology Microbiological Examination AFB staining $\square$ Fungal (KOH) staining $\square$ Culture and/or sensitivity

All these examinations were performed by a single trained specialist from respective department of our institute. Reports were followed up and archived as study document.

Statistical analysis The data analysis was performed using statistical software SPSS version 15.0. Categorical data was presented with frequencies \& percentages. Descriptive statistics were used to summarize the quantitative variables. Yield of each procedure was determined.

\section{RESULTS}

In 101 cases of various respiratory complaints included in our study, mean age of the population was $47.3 \pm 18.6$ years and age distribution was as shown in table 1 .
$65.34 \%$ patients were males and $34.65 \%$ were females. $55.44 \%$ patients from rural and $44.55 \%$ were from urban background. Cough and fever (90.09\% each) were most common symptoms followed by breathlessness $(59.40 \%)$, chest pain $(52.47 \%)$, weight loss $(22.77 \%)$ and haemoptysis $(14.85 \%)$. Other symptoms were change in voice, weakness and others as shown in figure 2.

Table 2 shows the lung zone involvement in study patients. Right lung was most commonly involved (middle, upper, and lower zones - 31.7\%, 21.8\% and $21.8 \%$ respectively) followed by left lung (middle, upper and lower zones $-17.8 \%, 12.9 \%$ and $18.8 \%$ respectively). Bilateral involvement was seen lesser number of patients with upper, middle, and lower zones involved in 6.9\%, $11.9 \%$ and $9.9 \%$ respectively

Figure 3 shows that 36.6 patients were smokers and $24.8 \%$ were alcoholic. Tobacco chewing was seen in $42.6 \%$ cases.

Consolidation $(48.5 \%)$ was most common feature followed by collapse (16.8\%), nodular shadow and mass lesion (12.9\% each) and hilar involvement $(10.9 \%)$. Other features were infiltration, cavity, cystic shadows and reticulonodular shadows. In patients with sputum for bacterial culture, $K$. pneumoniae (10.9\%) was frequently isolated, followed by $S$. pneumoniae $(6.9 \%), P$. aeruginosa $(4.0 \%)$ and E. coli $(3.0 \%)$.

Figure 4 shows that $92.1 \%$ patients had normal vocal cords whereas fixed left cord $(6.9 \%)$ and nodule on left vocal cord $(2.0 \%)$ were abnormal finding. No abnormality was seen in $50.5 \%$ and $65.3 \%$ right and left bronchi respectively. In right and left bronchi, purulent secretions were most common abnormality (34.7\% and $20.8 \%$ ), followed by nodular lesion (6.9\% and $4.0 \%)$, Congestion/ hyperaemia $(5.9 \%$ and $5.0 \%)$, growth $(5.0 \%$ and $8.9 \%$ ). Findings of bronchial wash were obtaned. On culture of wash, growth was seen in $32.1 \%$ with S. pneumoniae $(23.1 \%)$ being most common isolated organism. Acid-fast bacilli yield was $24.8 \%$ which was obtained after assessment using different methods. Fungal stain was positive in one patient only. Cytology reported inflammatory smear 
(62.0\%) as most common finding followed by malignancy (16.9\%). Fungal elements and features suggestive of tuberculosis were evident two and one patients respectively.

Findings of bronchial wash obtained from patients. On culture of wash, growth was seen in $32.1 \%$ with S. pneumoniae $(23.1 \%)$ being most common isolated organism.

Brush cytology yield was $73.3 \%$ for malignancy.

On histopathological examination of endobronchial biopsy specimen, Malignancy was seen in $65.5 \%$ (of these $52.6 \%$ were adenocarcinoma and $47.4 \%$ were squamous cell carcinoma). Features of suggestive of TB were reported in $2.5 \%$ cases.
Table 3 described final diagnosis after evaluation with bronchoscopy. Pulmonary TB was most common $(24.8 \%)$ followed by bacterial pneumonia (24.8\%), and malignancy (23.8\%). Other diagnoses included fungal pneumonia, ILD, and therapeutic for mucus plug. Bronchoscopy was non-diagnostic in $22.8 \%$ cases.

Table 1: Age distribution in study population

\begin{tabular}{|l|c|c|}
\hline Age & Frequency & Percentage \\
\hline $18-30$ & 29 & 28.71 \\
\hline $30-50$ & 29 & 28.71 \\
\hline $50-70$ & 31 & 30.69 \\
\hline $70-85$ & 12 & 11.88 \\
\hline Total & 101 & 100.0 \\
\hline
\end{tabular}

Figure 2: Clinical symptoms in study population

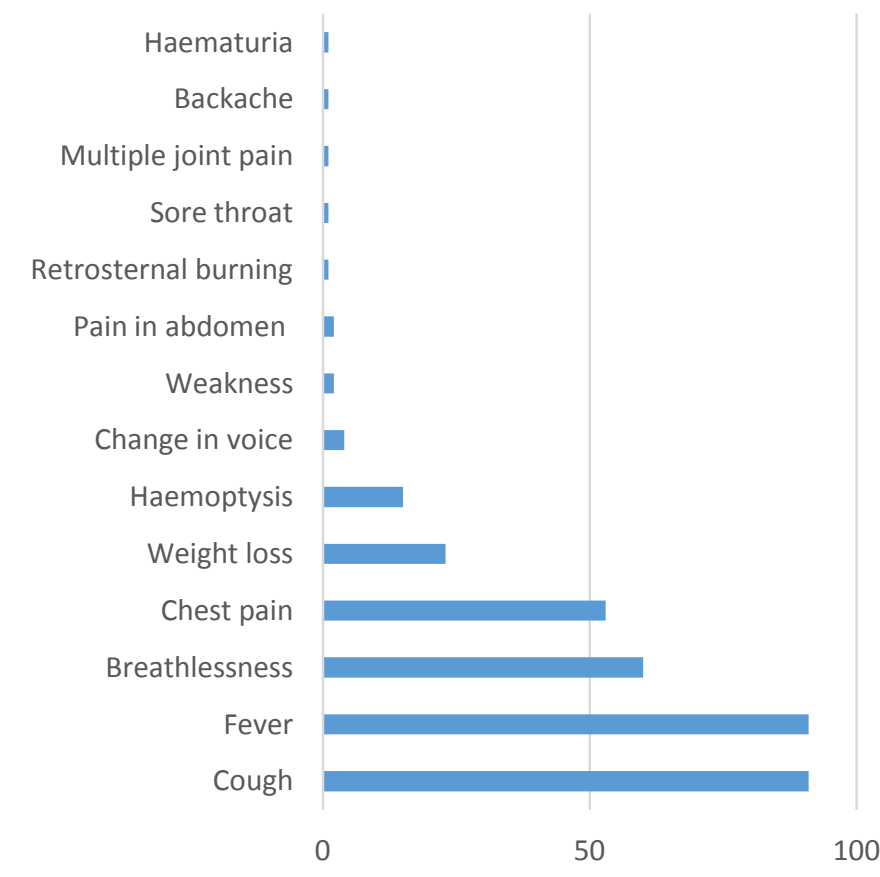

Table 2: Lung zone involvement by lesion as seen on chest $\mathrm{x}$-ray

\begin{tabular}{|l|c|c|}
\hline Lung Zone Involved & Frequency & Percentage \\
\hline Right - Upper & 22 & 21.8 \\
\hline Right - Middle & 32 & 31.7 \\
\hline Right - Lower & 22 & 21.8 \\
\hline Left - Upper & 13 & 12.9 \\
\hline Left - Middle & 18 & 17.8 \\
\hline Left - Lower & 19 & 18.8 \\
\hline Both Lungs - Upper & 7 & 6.9 \\
\hline Both Lungs - Middle & 12 & 11.9 \\
\hline Both Lungs - Lower & 10 & 9.9 \\
\hline
\end{tabular}


Figure 3: Personal habits in study population

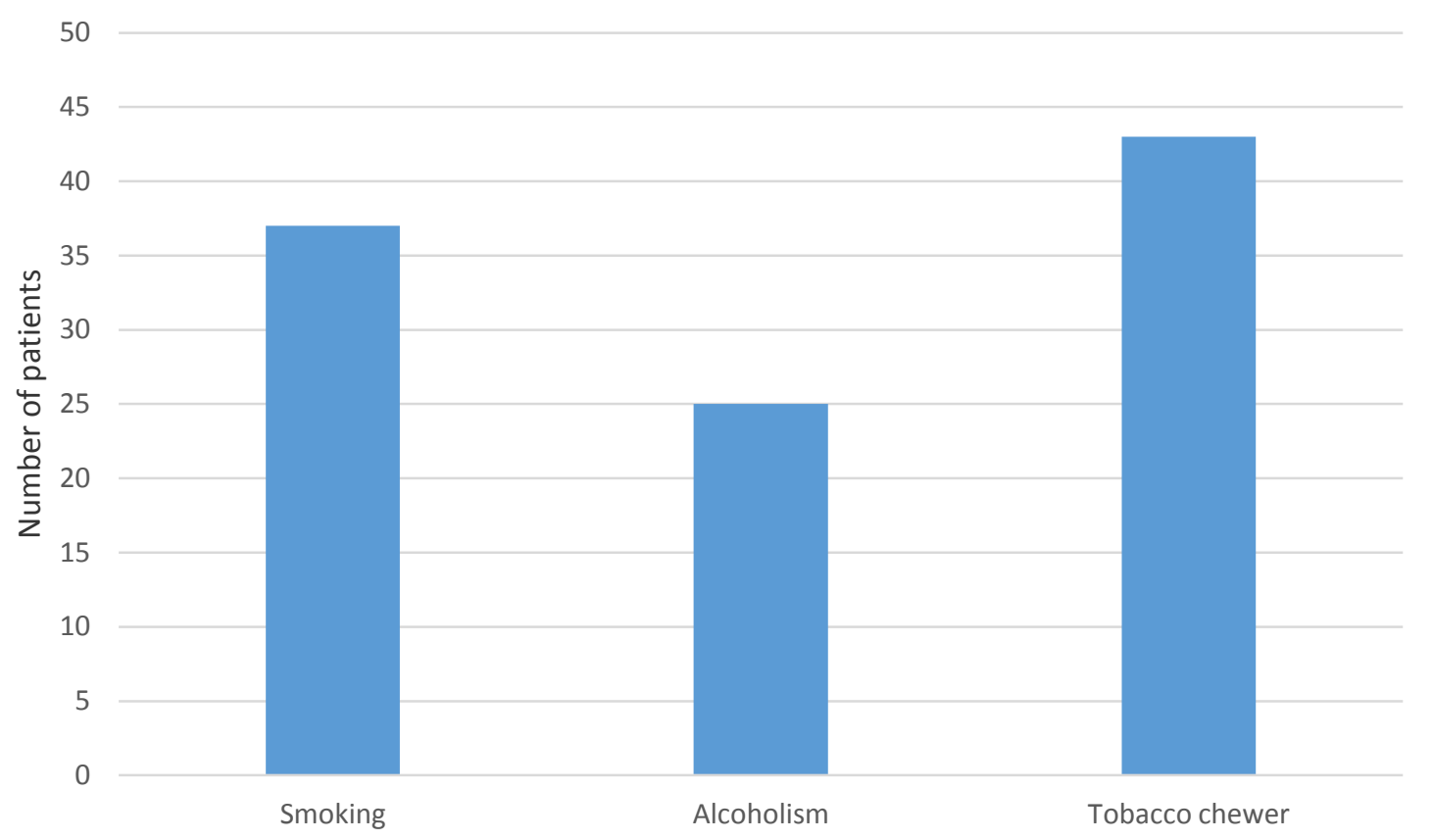

Figure 4: Condition of vocal cords visualized at bronchoscopy

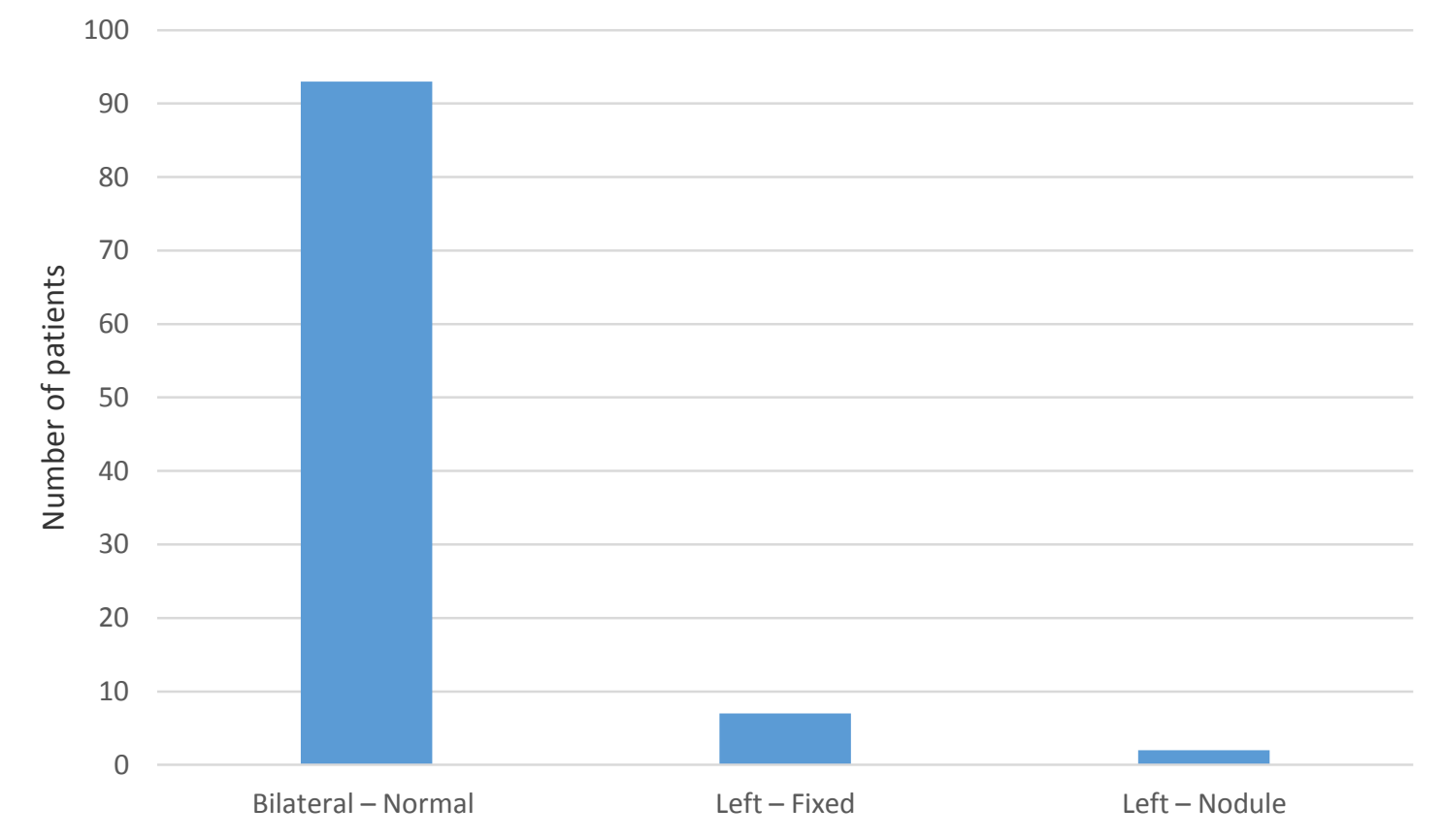

Table 3: Final diagnosis after bronchoscopy evaluation

\begin{tabular}{|l|c|c|}
\hline Diagnosis & Frequency & Percentage \\
\hline Pulmonary tuberculosis & 25 & 24.8 \\
\hline Malignancy & 24 & 23.8 \\
\hline Bacterial pneumonia & 25 & 24.8 \\
\hline Fungal pneumonia & 2 & 2.0 \\
\hline Interstitial lung disease & 1 & 1.0 \\
\hline Therapeutic for mucus plug & 1 & 1.0 \\
\hline Non-diagnostic after bronchoscopy & 23 & 22.8 \\
\hline
\end{tabular}




\section{DISCUSSION}

Bronchoscopy is one of the gold standard investigations in lung diseases. Tracheobronchial tree is directly visualized with Bronchoscopy. The discovery of flexible bronchoscopy was landmark in respiratory medicine in 1966 . Since then the utility of Flexible bronchoscopy has grown with gradual improvements in the techniques and expansion of indications from diagnostic to therapeutic ones. Changing patterns of lung malignancy in Indian setting makes bronchoscopy more useful for diagnostic and therapeutic interventions.47-49 We analysed 101 patients of various respiratory complaints and radiological features who underwent bronchoscopy for further evaluation.

Among various age groups, most patients were in age group of 50 to 70 years $(30.69 \%)$ followed by $28.71 \%$ each in 18 to 30 years and 30 to 50 year category. Only $11.88 \%$ were over 70 years of age. Nearly 2/3rd were males and rest were females. Over half of participants $(55.4 \%)$ belonged to rural region. A study from Gupta et al $(2015)^{5}$ reported that males $(71.6 \%)$ were more frequently undergone flexible bronchoscopy. Mean age of males was 48.4 years and of females was 45.2 years. In another similar study, Kapse et al $(2016)^{7}$ reported that most patients $(69.64 \%)$ who underwent FOB were in 51 to 70 years age group followed by 30 to 50 years (23.21\%) and remaining $7.14 \%$ patients being over 70 years. Greater number of males underwent FOB suggesting higher rated respiratory illnesses probably due to smoking and outdoor environmental exposures than females. This was evident in our study as $22.77 \%$ and $20.79 \%$ patients were farmer and labourer by occupation respectively.

Cough and fever (90.09\% each) were most common symptoms in patients. Other common symptoms were breathlessness $(59.40 \%)$, chest pain $(52.47 \%)$, weight loss $(22.77 \%)$ and haemoptysis (14.85\%). Change in voice was a complaint in $3.96 \%$ cases. In a clinicopathological study of lung malignancy, Rawat et al (2009) ${ }^{8}$ reported that cough $(72.90 \%)$ followed by fever (58.12\%), haemoptysis $(25.12 \%)$, chest pain $(55.67 \%)$ and dyspnoea $(50.74 \%)$ were common symptoms.
Among comorbid conditions, diabetes mellitus $(22.8 \%)$ and hypertension (13.9\%) were common. $5.9 \%$ of patients each had COPD and a history of TB. Jain et al $(2015)^{9}$ reported that $25 \%$ patients had diabetes, 3.3\% had hypertension, $21.6 \%$ had COPD and $26.6 \%$ had history of pulmonary TB.

We observed that $36.6 \%$ cases were smokers and $42.6 \%$ were tobacco chewers. Percentage of patients with alcoholism was $24.8 \%$. Jain et al $(2015)^{9}$ reported smoking in $65 \%$ cases, tobacco chewing in $43.33 \%$ and alcohol use in $40 \%$ cases. These findings suggest that habits of smoking and tobacco are quite common in patients with pulmonary symptoms and need detailed evaluation while assessing clinical history of these habits.

\section{Radiological Examination}

Right lung was more frequently involved. There were $21.8 \%, 31.7 \%$ and $21.8 \%$ of the lesions observed in right upper, middle, and lower zones respectively. In left lung, frequency of lesions in these zones was $12.9 \%, 17.8 \%$ and $18.8 \%$ respectively. When we looked at both lung involvement, middle zone had most frequent lesions (11.9\%) followed by lower (9.9\%) and upper (6.9\%) zone. Knowing site of lesion aids in clinical suspicion of a diagnosis. Exclusive upper zone involvement may suggest pulmonary tuberculosis, whereas lower zone may suggest penumonia. Rawat et al (2009) ${ }^{8}$ reported right lung involvement in majority of patients $(57.64 \%)$ followed by left lung involvement $(39.41 \%)$ and bilateral involvement in $2.96 \%$. These finding are similar to our observation.

Bhairagya et al $(2013)^{10}$ reported that in patients suspected of TB radiologically, an exclusive upper zone involvement was seen in $67 \%$ of patients while $16 \%$ of other patients had multi-lobar involvement including upper zones. In a study of bilateral lower zone shadows, Sherwani et al $(2005)^{11}$ reported that suspected diagnoses were TB, pneumonia, malignancy, bronchiectasis, ILD, among others. In combination with clinical profile, the radiological diagnosis was confirmed. The study still suggested that FNAC and fibreoptic bronchoscopy are of high 
value for pathological and microbiological confirmation of the diagnosis.

We observed consolidation as most common lesion $(48.5 \%)$ on $x$-ray chest followed by collapse (16.8\%), nodular shadow and mass lesion (12.9\% each), hilar involvement (10.9\%), infiltrative lesion $(9.9 \%)$ and cavity $(6.9 \%)$. Less frequent lesions were cystic shadows, reticulonodular shadows, fibrosis lesion and air-fluid level.Gupta et al (2015) reported that most common indication for performing bronchoscopy was malignancy (32.2\%) followed by infections $(18.8 \%)$, interstitial lung disease $(11 \%)$, haemoptysis $(11 \%)$, pleural effusion (3.6\%), mediastinal lymphadenopathy and hoarseness of voice $(20.1 \%)$, foreign body extraction $(0.26 \%)$.

Rawat et al (2009) ${ }^{9}$ reported mass lesion (46.31\%) as most common xray finding followed by collapseconsolidation in $40.89 \%$. Pleural effusion was reported in $4.43 \%$ cases. Among indications for FOB.

We performed CT in suspected cases. In 30 cases of chest CT, mass lesion (43.3\%) was most common followed by lymph node enlargement (40\%), consolidation (16.7\%), nodular shadow (13.3\%) and others. Performing CT before or after FOB is a dilemma. The clinical evidence remains unclear as to the timing of each procedure for diagnosing pulmonary lesions. Performing CT before bronchoscopy is suggested as it can help bronchoscopist understand the pathology well and bronchoscope can be directed in better way. ${ }^{[12]}$ Bronchoscopy

Normal vocal cords $(92.1 \%)$ were seen in majority. Abnormalities included fixed left fixed cord (6.9\%) and nodule on left vocal cord (2.0\%).Singh et al $(2008)^{13}$ reported vocal cord abnormalities in $7.2 \%$ cases in their study.

In majority of cases, no abnormal findings were seen in right $(50.5 \%)$ and left $(65.3 \%)$ bronchi. Presence of higher quantity of endo-bronchial secretions were most common abnormality $34.7 \%$ in right and $20.8 \%$ in left bronchus). Nodular lesion, congestion, growth, stenosis were common findings in both bronchi. A growth suspects underlying
malignancy.Increased secretions are suggestive of infective etiology.Singh et al $(2008)^{13}$ reported growth as most common finding (32\%) followed by purulent secretions (7\%), carbon deposits $(6.5 \%)$ and hypertrophied mucosa $(4.4 \%)$. They reported normal bronchoscopy in $30 \%$ cases.

Jain et al $(2015)^{9}$ reported normal bronchoscopy in $33.33 \%$ cases when performed for suspected cases of pulmonary TB.

To aid the diagnosis of findings at bronchoscopy, we performed bronchial wash. With culture of secretion in 78 cases, no growth was evident in 2/3rd cases. In rest cases, $S$. pneumoniae was most common $(23.1 \%)$ isolated organism followed by $\mathrm{K}$. pneumoniae $(7.7 \%)$ and P. aeruginosa $(1.3 \%)$. Yield for AFB was $24.8 \%$ when wash material was assessed using different techniques for diagnosis (ZN staining, GeneXpert and BACTEC MGIT 960 culture). Fungal stain was positive in one case only. Mohamed et al $(2013)^{14}$ reported that pneumonia was suspected in 357 (9.8\%) patients. Bacterial pneumonia was diagnosed microbiologically in 173 $(48.5 \%), P$. jiroveci pneumonia in $5(1.4 \%)$, and Candida species in $28(7.8 \%)$ patients. In $18(5 \%)$ patients, an lternative diagnosis was confirmed, mainly TB and 5 patients had interstitial lung disease (ILD). In the remaining 133 (37.3\%) samples, no organisms were isolated. Joos et al $(2006)^{15}$ reported that from suspected 37 cases of PTB, 10 were found positive by bronchoscopy.

In 71 cases of bronchial wash cytology, Inflammatory cytology (62\%) was most frequent in our study whereas malignancy was evident in $16.9 \%$ amongst which adenocarcinoma was seen in 5.6\% whereas poorly differentiated cytology was observed in $11.3 \%$ cases. Bronchial wash Cytology was non-conclusive in $16.9 \%$ whereas one case was found to be tubercular and fungal elements were reported in two cases. From among 15 cases of bronchial brush cytology, malignancy was seen in $73.3 \%$ cases. Rao et al $(1997)^{16}$ reported that bronchial wash cytology was inflammatory in $17.2 \%$ cases, normal in $10.3 \%$ cases, malignancy in $34.4 \%$ whereas suspicious of malignancy in $6.8 \%$ cases and negative for malignancy in $31.0 \%$ cases. 
We obtained biopsy specimens in 29 cases at bronchoscopy. $65.5 \%$ of these biopsies showed malignancy of which $52.6 \%$ was adenocarcinoma and $47.4 \%$ squamous cell carcinoma. Biopsy was inconclusive in $31 \%$ cases whereas AFB was confirmed in one case. In a study assessing value of BAL in diagnosing peripheral lung malignancies, Gracia et al (1992) ${ }^{17}$ performed needle aspiration or lung biopsy in case of inconclusive results on BAL and brushing. In 55 patients diagnosed to have malignant disease (23 adenocarcinomas, 22 squamous cell carcinomas, six small cell carcinomas, and four bronchioloalveolar cell carcinomas).

Transbronchial lung biopsy (TBLB) was performed in 5 cases in which it was non-conclusive in 3 cases whereas one case each of interstitial lung disease and malignancy was diagnosed. Trans-bronchial needle aspiration (TBNA) in four cases revealed malignancy ( 3 adenocarcinoma and one squamous cell carcinoma) giving $100 \%$ yield.

In a study of TBLB, Clark et al $(1977)^{18}$ reported diagnosis of cryptogenic fibrosing alveolitits (15/25), sarcoidosis (4/7), Pneumoconiosis (4/8), carcinomatosis (8/9). Yield for diffuse lesions was $62 \%$ with TBLB.

The bronchial brushing was positive for malignant cells in $61.53 \%$, washing in $70.76 \%$, while endobronchial biopsy was positive in $76.92 \%$. With use of these procedures at bronchoscopy, diagnosis of suspected pulmonary lesions is possible. Definite diagnosis of lesions in our patients was possible in 88 cases giving yield of $87.2 \%$. Major diagnoses were pulmonary tuberculosis $(24.8 \%)$, bacterial pneumonia (24.8\%), and malignancy (23.8\%). Rare diagnoses were fungal pneumonia in 2 cases, ILD in 1 case, and therapeutic use for dislodging mucous plug in 1 case. In a similar study, Mohamed et al $(2013)^{14}$ diagnostic utility of FOB to be $67 \%$. Indication wise yield was $70 \%$ in malignancy, $48.5 \%$ in infections, $58.5 \%$ in TB, $44 \%$ in haemoptysis evaluation, $47 \%$ in abnormal radiological finding and $36 \%$ in other. Thus, bronchoscopy aided by various methods like washing, brushing, and biopsy helps in diagnosis of common as well as rare pathologies

of

lungs.

\section{CONCLUSIONS}

Patients of respiratory complaints with lesion visible on chest $\mathrm{x}$-ray who are undiagnosed can derive benefit by flexible bronchoscopy. Use of supportive techniques of bronchial wash, brushing, biopsy aid in diagnosis.

We observed good yield of wash in diagnosing various pulmonary lesion. A malignancy inconclusive in wash cytology was further diagnosed using biopsy samples especially in mass lesions seen on bronchoscopy. Brushing cytology provided yield of over $70 \%$ for diagnosis of malignancy whereas endobronchial biopsy yield was $65.5 \%$ for malignancy.

TBLB and TBNA yield was $40 \%$ and $100 \%$ respectively for diagnosis of patients which demand further caution as these methods involved small number of patients.

\section{REFERENCES}

1. Ohata M. History and Progress of Bronchology in Japan.Bronchology - Japan Society for Bronchology. 1919;26:539-546.

2. Herth F. Bronchoscopic techniques in diagnosis and staging of lung cancer. Breathe. 2011;7(4):324-337.

3. Fuladi AB, Munje RP, Tayade BO. Value of washings and brushings, And Biopsy at fibreoptic bronchoscopy in the diagnosis of lung cancer. Journal, Indian Academy of Clinical Medicine.2004;5(2):137-142.

4. Bhadke B, Munje R, Mahadani J, Surjushe A, Jalgaonkar P. Utility of fiberoptic bronchoscopy in diagnosis of various lung conditions : Our experience at rural medical college.Lung India. 2010;27(3):118-121.

5. Gupta A.A, Sehgal IS, Dhooria S, Singh N, Aggarwal AN, Gupta D, et al. Indications for performing flexible bronchoscopy: Trends over 34 years at a tertiary care hospital. Lung India. 2015;32(3):211-215.

6. Garg B, Sood N, Sidhu UP, Malhotra V. Role of Fiberoptic Bronchoscopy and Utility 
of Bronchial Washings and Brushings in the Diagnosis of Lung Diseases.Indian J Chest Dis Allied Sci 2013;55:145-148.

7. Kapse VR, Mhaisekar DG, Totewad D, Kulkarni M. Study of comparison of yield of bronchial brushing and broncho-alveolar lveolar lavage in diagnosis of lung cancer.International Journal of Recent Trends in Science and Technology. 2016;19(1):69-72

8. Rawat J, Sindhwani G, Gaur D, Dua R, Saini S. Clinico-pathological profile of lung cancer in Uttarakhand. Lung India.2009; 26(3):74-6

9. Jain S RK, J AB, K PG. Role of fiberoptic bronchoscopy in patients with sputum smear negative for acid fast bacilli and chest $\mathrm{x}$ - ray suggestive of pulmonary tuberculosis. Journal of Evidence based Medicine and Healthcare.2015;2(2):155-164.

10. Bairagya TD, Biswas S, Das A, Das SK, Sinha A. The role of induced sputum in the diagnosis of pulmonary tuberculosis. Lung India.2013;30(3):199-202

11. Sherwani R, Akhtar K, Siddiqui T, Bhargava $\mathrm{R}$, Zaheer S. Bilateral lower zone shadows on chest radiograph - A clinicopathological profile.Indian J Tuberc 2005; 52:137-142.

12. Høiby N, Koch C. Maintenance treatment of chronic Pseudomonas aeruginosa infection in cystic fibrosis. Thorax. 2000;55:349-350.

13. Singh R, Kaur H, Singh G. Dignostic yield of fiberoptic bronchoscopy in a teaching hospital. JK Sci. 2008;10(4):178-180

14. Mohamed SAA, Metwally MMA, Abd ElAziz NMA, Gamal Y. Diagnostic utility and complications of flexible fiberoptic bronchoscopy in Assiut University Hospital: A 7-year experience. The Egyptian Society of Chest Diseases and Tuberculosis. 2013;62(3):535-540.

15. Joos L, Patuto N, Chhajed PN, Tamm M. Diagnostic yield of flexible bronchoscopy in current clinical practice. Swiss Med Wkly. 2006;136:155-159
16. Rao S, Rao S, Lal A, Barathi G, Dhanasekar T, Duvuru P. Bronchial wash cytology: A study on morphology and morphometry. J Cytol.2014;31(2):63-67.

17. Gracia JDE, Bravo C, Miravitlles M, Tallada N, Orriols R,Bellmunt J, et al. Diagnostic Value of Bronchoalveolar Lavage in Peripheral Lung Cancer. Am Rev Respir Dis. 1993; 147:649-652.

18. Clark RA, Gray PB, Townshend RH, Howard P. Transbronchial lung biopsy: a review of 85 cases. Thorax.1977;32:546549. 